\title{
Polarized-VAE: Proximity Based Disentangled Representation Learning for Text Generation
}

\author{
Vikash Balasubramanian ${ }^{1 *}$ Ivan Kobyzev², Hareesh Bahuleyan², \\ Ilya Shapiro ${ }^{3}$, Olga Vechtomova ${ }^{1}$ \\ ${ }^{1}$ University of Waterloo, ${ }^{2}$ Borealis AI, ${ }^{2}$ University of Windsor \\ \{v9balasu, ovechtom\} @uwaterloo.ca \\ ivan.kobyzev@borealisai.com \\ hareeshbahuleyan@gmail.com \\ ishapiro@uwindsor.ca
}

\begin{abstract}
Learning disentangled representations of realworld data is a challenging open problem. Most previous methods have focused on either supervised approaches which use attribute labels or unsupervised approaches that manipulate the factorization in the latent space of models such as the variational autoencoder (VAE) by training with task-specific losses. In this work, we propose polarized-VAE, an approach that disentangles select attributes in the latent space based on proximity measures reflecting the similarity between data points with respect to these attributes. We apply our method to disentangle the semantics and syntax of sentences and carry out transfer experiments. PolarizedVAE outperforms the VAE baseline and is competitive with state-of-the-art approaches, while being more a general framework that is applicable to other attribute disentanglement tasks.
\end{abstract}

\section{Introduction}

Learning representations of real-world data using deep neural networks has accelerated research within a number of fields including computer vision and natural language processing (Zhang et al., 2018). Previous work has advocated for the importance of learning disentangled representations (Bengio et al., 2013; Tschannen et al., 2018). Although attempts have been made to formally define disentangled representations (Higgins et al., 2018), there is no widely accepted definition of disentanglement. However, the general consensus is that a disentangled representation should separate the distinct factors of variation that explain the data (Bengio et al., 2013). Intuitively, a greater level of interpretability can be achieved when independent latent units are used to encode different attributes of the data (Burgess et al., 2018).

However, recovering and separating all the distinct factors of variation in the data is a challenging problem. For real-world datasets, there may not be a way to separate each factor of variation into a single dimension in the learnt fixed-size vector representation. An easier problem would be to separate complex factors of interest into distinct subspaces of the learnt representation. For instance, a representation for text could be separated into content and style subspaces, which then enables style transfer.

Unsupervised disentanglement of underlying factors using variational autoencoders (Kingma and Welling, 2014) has been explored in previous work (Higgins et al., 2017; Kim and Mnih, 2018). However, Locatello et al. (2019) argue that completely unsupervised disentanglement of the underlying factors may be impossible without supervision or inductive biases. Disentangling textual attributes in a completely unsupervised manner has been shown to be especially difficult, but attempts have been made to leverage it for controllable text generation (Xu et al., 2020).

In this work, we propose an approach referred to as polarized-VAE ${ }^{1}$ to disentangle the latent space into subspaces corresponding to different factors of variation. We control the relative location of representations in a particular latent subspace based on the similarity of their respective input data points according to a defined criterion (that corresponds to an attribute in the input space, e.g., syntax). This encourages similar points to be grouped together and dissimilar points to be farther away from each other in that subspace. Figuratively, we polarize the latent subspaces, and hence the name.

Most previous work on supervised disentanglement for text has focused on adversarial training (John et al., 2019; Yang et al., 2018). Recently, the task of disentangling textual semantics and syntax into distinct subspaces has received attention

\footnotetext{
${ }^{1}$ The code is available at https://github.com/ vikigenius/prox_vae
} 
from researchers. For instance, Chen et al. (2019b) use a sentence VAE model with several multitask losses such as paraphrase loss and word position loss for this disentanglement task. Bao et al. (2019) incorporate adversarial training and make use of syntax trees along with specific multitask losses to disentangle semantics and syntax.

In polarized-VAE, we achieve disentanglement through distance based learning. In contrast to previous approaches, our method does not require the use of several multitask losses or adversarial training, both of which can result in optimization challenges. Furthermore, we do not need precise attribute labels, and we show that using proxy labels based on the concept of similarity is sufficient.

In summary, the main contributions of this paper are three-fold: (1) We propose a general framework for learning disentangled representations. Even though we test our method on an NLP task, the underlying concept is very general and can be applied to other domains such as computer vision; (2) We provide a method for disentanglement that does not rely on adversarial training or specialized multitask losses; (3) We demonstrate an application of our method by disentangling the latent space into subspaces corresponding to syntax and semantics. Such a setting can be used to perform controlled text decoding such as generating a paraphrase with a desired sentence structure.

\section{Proposed Approach}

In VAEs, a probabilistic encoder $q_{\phi}(\boldsymbol{z} \mid \boldsymbol{x})$ is used to encode a sentence $\boldsymbol{x}$ into a latent variable $\boldsymbol{z}$, and a probabilistic decoder $p_{\theta}(\boldsymbol{x} \mid \boldsymbol{z})$ attempts to reconstruct the original sentence $\boldsymbol{x}$ from its latent representation $\boldsymbol{z}$. The objective is to minimize the following loss function:

$$
\mathcal{L}_{\text {vae }}=\mathcal{L}_{\text {rec }}+\lambda_{\text {kl }} \mathcal{L}_{\mathrm{kl}}
$$

where $\mathcal{L}_{\text {rec }}=-\mathbb{E}_{q_{\phi}(\boldsymbol{z} \mid \boldsymbol{x})}\left[\log p_{\theta}(\boldsymbol{x} \mid \boldsymbol{z})\right]$ is the sentence reconstruction loss and $\mathcal{L}_{\mathrm{kl}}=$ $\mathcal{D}_{\mathrm{kl}}\left(q_{\phi}(\boldsymbol{z} \mid \boldsymbol{x}) \| p(\boldsymbol{z})\right) \quad$ is the Kullback-Leibler (KL) divergence loss. The KL term ensures that the approximate posterior $q_{\phi}(\boldsymbol{z} \mid \boldsymbol{x})$ is close to the prior $p(\boldsymbol{z})$, which is typically assumed to be the standard normal $\mathcal{N}(\mathbf{0}, \mathbf{I}) ; \lambda_{\mathrm{kl}}$ is a hyperparameter that controls the extent of KL regularization.

The idea behind our polarized-VAE approach is to impose additional proximity regularization on the latent subspaces learnt by VAEs. Let $C=\left\{c_{1}, \ldots, c_{k}\right\}$ be the collection of criteria, based on which we wish to disentangle the latent space $z$ of the VAE into $k$ subspaces: $\boldsymbol{z}=\left[\boldsymbol{z}^{(1)}, \ldots, \boldsymbol{z}^{(k)}\right]$. Here $\boldsymbol{z}^{(i)}$ denotes the latent subspace corresponding to the criterion $c_{i}$ (see Figure 1). In this paper, we focus on the case where the latent space is disentangled into semantics $\left(c_{1}\right)$ and syntax $\left(c_{2}\right)$, i.e., $k=2$.

\subsection{Supervision based on Similarity}

We assume that we have information (possibly noisy) about pairwise similarities of the input sentences. Given a pair of sentences, the similarity information can be either a binary label (whether both the sentences belong to the same class or not) or an integer or continuous scalar variable (e.g., edit distance). In this work, the similarity criterion is a binary label:

$\operatorname{Sim}\left(\boldsymbol{x}_{i}, \boldsymbol{x}_{j} \mid c\right)= \begin{cases}1, & \text { if } \boldsymbol{x}_{i} \text { and } \boldsymbol{x}_{j} \text { are similar } \\ & \text { w.r.t. the criterion } c \in C \\ 0, & \text { otherwise }\end{cases}$

In our case, the two criteria for disentanglement are semantics $\left(c_{1}\right)$ and syntax $\left(c_{2}\right)$. We use this additional information to regularize the latent space of the VAE by incorporating the proximity based loss functions, denoted as $D\left(\boldsymbol{z}_{i}^{(1)}, \boldsymbol{z}_{j}^{(1)} \mid c_{1}\right)$ and $D\left(\boldsymbol{z}_{i}^{(2)}, \boldsymbol{z}_{j}^{(2)} \mid c_{2}\right)$.

\subsection{Training Method and Proximity Function}

Extending the traditional VAE approach, we have a set of RNN-based encoders parameterized by $\phi_{c}$ that learn the approximate posteriors $q_{\phi_{c}}\left(\boldsymbol{z}^{(c)} \mid \boldsymbol{x}\right)$. Given two data points $\boldsymbol{x}_{i}$ and $\boldsymbol{x}_{j}$, we denote the proximity of their encodings in the latent subspace by $D\left(q_{\phi_{c}}\left(\boldsymbol{z}^{(c)} \mid \boldsymbol{x}_{i}\right), q_{\phi_{c}}\left(\boldsymbol{z}^{(c)} \mid \boldsymbol{x}_{j}\right)\right)$. We experiment with multiple forms of proximity functions and found cosine distance to perform the best:

$$
\begin{aligned}
D\left(q_{\phi_{c}}\left(\boldsymbol{z} \mid \boldsymbol{x}_{i}\right), q_{\phi_{c}}\left(\boldsymbol{z} \mid \boldsymbol{x}_{j}\right)\right) & =d_{c}\left(\boldsymbol{z}_{i}, \boldsymbol{z}_{j}\right) \\
& =\frac{1}{2}\left(1-\frac{\boldsymbol{z}_{i} \boldsymbol{z}_{j}}{\left\|\boldsymbol{z}_{i}\right\|\left\|\boldsymbol{z}_{j}\right\|}\right)
\end{aligned}
$$

Based on the above distance, we add a regularization term to the VAE loss function as follows. For each example $(\boldsymbol{x}, c)$, we have a positive sample $\boldsymbol{x}_{p}$ and $m$ negative samples $\boldsymbol{x}_{n_{1}}, \ldots, \boldsymbol{x}_{n_{m}}$, such that $\operatorname{Sim}\left(\boldsymbol{x}, \boldsymbol{x}_{p} \mid c\right)=1$ and $\operatorname{Sim}\left(\boldsymbol{x}, \boldsymbol{x}_{n_{j}} \mid c\right)=0 ; j \in$ $\{1, \ldots, m\}$ :

$\mathcal{L}_{c}=\max \left(0,1+d_{c}\left(\boldsymbol{z}, \boldsymbol{z}_{p}\right)-\frac{1}{m} \sum_{j=1}^{m} d_{c}\left(\boldsymbol{z}, \boldsymbol{z}_{n_{j}}\right)\right)$ 


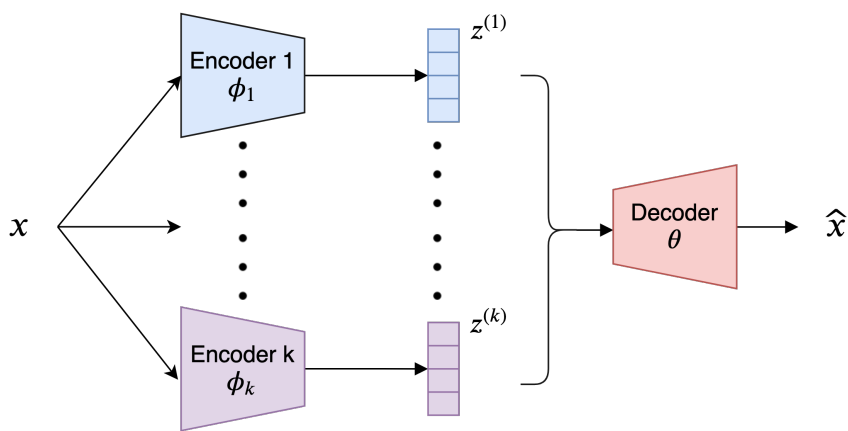

Figure 1: Model Architecture

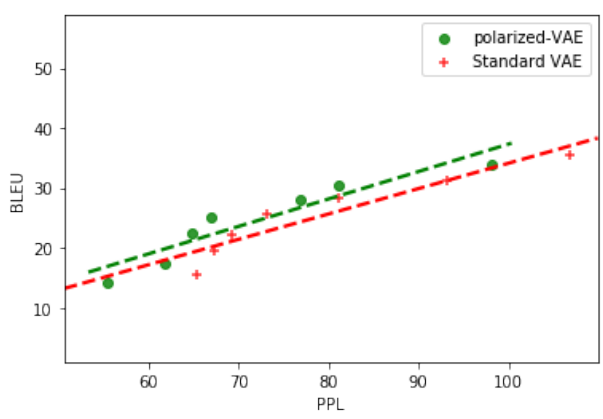

Figure 2: BLEU vs. PPL trade-off
This regularization function can be viewed as a max-margin loss over the proximity function. The final objective then becomes

$$
\mathcal{L}=\mathcal{L}_{\text {vae }}+\sum_{c \in C} \lambda_{c} \mathcal{L}_{c}
$$

\section{Experiments}

To demonstrate the effectiveness of polarized-VAE in obtaining disentangled representations, we carry out semantics-syntax separation of textual data, using the Stanford Natural Language Inference dataset (SNLI, Bowman et al. (2015)). Model implementation details are provided in Appendix A.

\subsection{Reconstruction and Sample Quality}

We evaluate our model on reconstruction and sample quality to ensure that the distance-based regularization used does not adversely impact its reconstruction or sampling capabilities. For this purpose, we compare our model and the standard VAE on two metrics: reconstruction BLEU (Papineni et al., 2002) and the Forward Perplexity (PPL) ${ }^{2}$ (Zhao et al., 2018) of the generated sentences obtained by sampling from the model's latent space. As seen in Figure 2, there is a clear trade-off between reconstruction quality and sample quality, which is expected. Overall, polarized-VAE performs slightly better than standard VAE and this indicates that the proximity-based regularization does not inhibit the model capabilities.

\subsection{Controlled Generation and Transfer}

We follow previous work (Chen et al., 2019a; Bao et al., 2019) and analyze the performance of controlled generation by evaluating syntax transfer in generated text. Given two sentences, $\boldsymbol{x}_{\mathrm{sem}}$ and $\boldsymbol{x}_{\mathrm{syn}}$, we wish to generate a third sentence that

\footnotetext{
2013)

${ }^{2}$ PPL is computed using the KenLM toolkit (Heafield et al.,
}

combines the semantics of $\boldsymbol{x}_{\mathrm{sem}}$ and the syntax of $\boldsymbol{x}_{\mathrm{syn}}$ using the following procedure:

$$
\begin{array}{cl}
\boldsymbol{z}_{\mathrm{sem}} \sim q_{\phi_{1}}\left(\boldsymbol{z}^{(1)} \mid \boldsymbol{x}_{\mathrm{sem}}\right) & ; \boldsymbol{z}_{\mathrm{syn}} \sim q_{\phi_{2}}\left(\boldsymbol{z}^{(2)} \mid \boldsymbol{x}_{\mathrm{syn}}\right) \\
\boldsymbol{z}=\left[\boldsymbol{z}_{\mathrm{sem}}, \boldsymbol{z}_{\mathrm{syn}}\right] & ; \boldsymbol{x} \sim p_{\theta}(\boldsymbol{x} \mid \boldsymbol{z})
\end{array}
$$

Following the evaluation methodology of Bao et al. (2019), we measure transfer based on (1) semantic content preservation for the semantic subspace and (2) the tree edit distance (Zhang and Shasha, 1989) for the syntactic subspace.

We consider pairs of sentences from the SNLI test set for evaluation. We would like the generated sentence to be close to $\boldsymbol{x}_{\mathrm{sem}}$ and different from $\boldsymbol{x}_{\mathrm{syn}}$ in terms of semantics, which is measured using BLEU scores. We also report the difference to indicate the strength of transfer denoted by $\triangle$ BLEU. Additionally, we would like the generated sentence to be syntactically similar to $\boldsymbol{x}_{\text {syn }}$ and different from $\boldsymbol{x}_{\mathrm{sem}}$, which is measured by averaged sentence-level Tree Edit Distance (TED). We also report $\triangle$ TED to indicate the strength of the syntax transfer. Finally, we use the Geometric Mean of $\triangle B L E U$ and $\triangle$ TED to report a combined score $\Delta \mathrm{GM}$.

Our default variant of polarized-VAE uses the entailment labels from SNLI dataset as a proxy for semantic similarity based on which positive and negative samples are chosen. For this model, we threshold the difference in TED of syntax parses as a proxy for syntactic similarity. As shown in Table 1, we also evaluate three other variants of our model. In polarized-VAE (wo) we use word overlap (BLEU scores) as a heuristic proxy for estimating semantic similarity, while keeping syntactic training unchanged. We also experiment with heuristics for syntax in polarized-VAE ( $l e n$ ) where we use length as a heuristic proxy for syntax, while still using ground truth entailment labels for semantic training. Finally we combine these two 


\begin{tabular}{|c|c|c|c|c|c|c|c|c|c|c|}
\hline Model & $\begin{array}{r}\mathbf{B I} \\
\boldsymbol{x}_{\mathrm{sem}}{ }^{\uparrow}\end{array}$ & $\begin{array}{l}\boldsymbol{x}_{\mathrm{syn}} \\
\downarrow\end{array}$ & $\Delta \mathrm{BLEU}^{\uparrow}$ & $\boldsymbol{x}_{\mathrm{sem}}{ }^{\uparrow}$ & $\begin{array}{l}\mathbf{D} \\
\boldsymbol{x}_{\mathrm{syn}}\end{array}$ & $\Delta \mathrm{TED}^{\uparrow}$ & $\Delta \mathrm{GM}^{\uparrow}$ & \multicolumn{2}{|c|}{ Human Eval (\%) } & $\begin{array}{l}\text { val (\%) } \\
\text { fluency }\end{array}$ \\
\hline Standard VAE & 4.75 & 4.67 & 0.08 & 13.70 & 13.60 & 0.10 & 0.28 & 11 & 11 & 43 \\
\hline Bao et al. (2019) & 13.74 & 6.15 & 7.59 & 16.19 & 13.10 & 3.08 & 4.83 & 24 & 58 & 19 \\
\hline polarizec & 10.78 & 0.92 & 9.86 & 14.09 & 11.67 & 2.42 & 4.88 & 65 & 31 & 38 \\
\hline larized- $\mathrm{V}$ & 9.82 & 0.84 & 8.98 & 14.12 & 11.65 & 2.47 & 4.71 & - & - & - \\
\hline polarized-VAE (len) & 10.10 & 0.76 & 9.34 & 12.68 & 11.44 & 1.44 & 3.67 & - & - & - \\
\hline polarized-VAE (wo, len) & 9.41 & 0.87 & 8.54 & 12.65 & 11.48 & 1.17 & 3.16 & - & - & - \\
\hline
\end{tabular}

Table 1: Syntax transfer results on SNLI. Bao et al. (2019) report TED after multiplying by 10, we report their score after correction. For each model, the human evaluation scores represent percentage of instances that it was ranked the best for a given criteria (semantics preservation/syntax transfer/fluency).

heuristics in polarized-VAE (wo, len), which can be viewed as an unsupervised variant that does not make use of any ground truth labels or syntax trees.

Our model outperforms the VAE baseline on all metrics. In comparison to (Bao et al., 2019), polarized-VAE is much better at ignoring the semantic information present in $\boldsymbol{x}_{\mathrm{syn}}$ during syntax transfer, as evidenced by our lower BLEU scores w.r.t. $\boldsymbol{x}_{\mathrm{syn}}$. On the other hand, we perform slightly worse on BLEU w.r.t. $\boldsymbol{x}_{\text {sem }}$. Our model does a better job at matching the syntax of sentence $\boldsymbol{x}_{\mathrm{syn}}$ as indicated by the lower TED score w.r.t. $\boldsymbol{x}_{\mathrm{syn}}$. Qualitative samples of syntax transfer are provided in Appendix C.

\subsection{Human Evaluation}

We carried out a human evaluation study for comparing outputs generated from different models. The test setup is as follows - we provide as input two sentences, $x_{\mathrm{sem}}$ and $x_{\mathrm{syn}}$ to the model; we wish to generate a sentence that combines the semantics of $x_{\mathrm{sem}}$ and the syntax of $x_{\mathrm{syn}}$. We asked 5 human annotators to evaluate the outputs from the 3 models: baseline-VAE, polarized-VAE and the model from (Bao et al., 2019).

Each annotator was shown the input sentences $\left(x_{\mathrm{sem}}\right.$ and $\left.x_{\mathrm{syn}}\right)$ and the outputs from the 3 models (randomized so that the evaluator is unaware of which output corresponds to which model). They were then asked to pick the one best output for each of the following three criteria: (1) semantic preservation - level of semantic similarity with respect to $x_{\text {sem }}$, (2) syntactic transfer - level of syntactic similarity with respect to $x_{\text {syn }}$ and (3) fluency. We obtained annotations on 100 test set examples from SNLI dataset. To aggregate the annotations, we used majority voting with manual tie breaking to find the best model for each test example (and for each test criteria).

For each model, we report the percentage of instances where it was voted as best for each criteria.
From the human evaluation results in Table 1, we note that polarized-VAE is better at semantic transfer and worse at syntactic transfer in comparison to (Bao et al., 2019). The human evaluation results are consistent with the automatic evaluation metrics, where polarized-VAE scores higher on $\triangle \mathrm{BLEU}$ (indicator of semantic transfer strength) and (Bao et al., 2019) is better at $\triangle$ TED (indicator of syntax transfer strength). With respect to fluency criterion, polarized-VAE ranks higher than (Bao et al., 2019). However, the most fluent sentences are produced by the baseline VAE. We hypothesise this to be due to the presence of additional regularization terms in the loss functions of both (Bao et al., 2019) and polarized-VAE, which in turn affects the fluency of their generated text (due to the deviation from the reconstruction objective).

\section{Conclusion and Future Work}

We proposed a general approach for disentangling latent representations into subspaces using proximity functions. Given a pair of data points, a predefined similarity criterion in the original input space determines their relative distance in the corresponding latent subspace, which is modelled via a proximity function. We apply our approach to the task of disentangling semantics and syntax in text. Our model substantially outperforms the VAE baseline and is competitive with the state-of-theart approach while being more general as we do not use specific multitask losses or architectures to encourage preservation of semantic or syntactic information. Our methodology is orthogonal to the multitask learning approaches by Chen et al. (2019b) and Bao et al. (2019) and can be naturally combined with their methods. We would further like to investigate this approach on disentanglement applications outside of NLP. Another interesting research direction would be to further explore suitable proximity functions and identify their properties that could facilitate disentanglement. 


\section{Acknowledgements}

We would like to thank Dr. Pascal Poupart for his valuable insights and ideas. We would also like to thank Compute Canada (www. computecanada.ca) for their support and GPU resourcees.

This Research was funded by the MITACS Accelerate program in collaboration with Borealis AI.

\section{References}

Hareesh Bahuleyan. 2018. Natural language generation with neural variational models. CoRR, abs/1808.09012.

Yu Bao, Hao Zhou, Shujian Huang, Lei Li, Lili Mou, Olga Vechtomova, Xinyu Dai, and Jiajun Chen. 2019. Generating sentences from disentangled syntactic and semantic spaces. In Proceedings of the 57th Annual Meeting of the Association for Computational Linguistics, pages 6008-6019.

Yoshua Bengio, Aaron Courville, and Pascal Vincent. 2013. Representation learning: A review and new perspectives. IEEE transactions on pattern analysis and machine intelligence, 35(8):1798-1828.

Samuel Bowman, Gabor Angeli, Christopher Potts, and Christopher D Manning. 2015. A large annotated corpus for learning natural language inference. In Conference on Empirical Methods in Natural Language Processing, EMNLP 2015, pages 632-642. Association for Computational Linguistics (ACL).

Samuel R. Bowman, Luke Vilnis, Oriol Vinyals, Andrew Dai, Rafal Jozefowicz, and Samy Bengio. 2016. Generating sentences from a continuous space. Proceedings of The 20th SIGNLL Conference on Computational Natural Language Learning.

Christopher P. Burgess, Irina Higgins, Arka Pal, Loïc Matthey, Nick Watters, Guillaume Desjardins, and Alexander Lerchner. 2018. Understanding disentangling in $\beta$-vae. CoRR, abs/1804.03599.

Mingda Chen, Qingming Tang, Sam Wiseman, and Kevin Gimpel. 2019a. Controllable paraphrase generation with a syntactic exemplar. In Proceedings of the 57th Annual Meeting of the Association for Computational Linguistics, pages 5972-5984, Florence, Italy. Association for Computational Linguistics.

Mingda Chen, Qingming Tang, Sam Wiseman, and Kevin Gimpel. 2019b. A multi-task approach for disentangling syntax and semantics in sentence representations. In Proceedings of the 2019 Conference of the North American Chapter of the Association for Computational Linguistics: Human Language Technologies, Volume 1 (Long and Short Papers), pages 2453-2464.
Kenneth Heafield, Ivan Pouzyrevsky, Jonathan H. Clark, and Philipp Koehn. 2013. Scalable modified Kneser-Ney language model estimation. In Proceedings of the 51st Annual Meeting of the Association for Computational Linguistics, pages 690-696, Sofia, Bulgaria.

Irina Higgins, David Amos, David Pfau, Sébastien Racanière, Loïc Matthey, Danilo J. Rezende, and Alexander Lerchner. 2018. Towards a definition of disentangled representations. CoRR, abs/1812.02230.

Irina Higgins, Loïc Matthey, Arka Pal, Christopher Burgess, Xavier Glorot, Matthew Botvinick, Shakir Mohamed, and Alexander Lerchner. 2017. beta-vae: Learning basic visual concepts with a constrained variational framework. In 5th International Conference on Learning Representations, ICLR 2017, Toulon, France, April 24-26, 2017, Conference Track Proceedings. OpenReview.net.

Sepp Hochreiter and Jürgen Schmidhuber. 1997. Long short-term memory. Neural computation, 9:173580

Vineet John, Lili Mou, Hareesh Bahuleyan, and Olga Vechtomova. 2019. Disentangled representation learning for non-parallel text style transfer. In Proceedings of the 57th Annual Meeting of the Association for Computational Linguistics, pages 424-434, Florence, Italy. Association for Computational Linguistics.

Hyunjik Kim and Andriy Mnih. 2018. Disentangling by factorising. In Proceedings of the 35th International Conference on Machine Learning, volume 80 of Proceedings of Machine Learning Research, pages 2649-2658, Stockholmsmässan, Stockholm Sweden. PMLR.

Diederik P. Kingma and Jimmy Ba. 2015. Adam: A method for stochastic optimization. In 3rd International Conference on Learning Representations, ICLR 2015, San Diego, CA, USA, May 7-9, 2015, Conference Track Proceedings.

Diederik P. Kingma and Max Welling. 2014. Autoencoding variational bayes. In 2 nd International Conference on Learning Representations, ICLR 2014, Banff, AB, Canada, April 14-16, 2014, Conference Track Proceedings.

Francesco Locatello, Stefan Bauer, Mario Lucic, Gunnar Rätsch, Sylvain Gelly, Bernhard Schölkopf, and Olivier Bachem. 2019. Challenging common assumptions in the unsupervised learning of disentangled representations. In Reproducibility in $\mathrm{Ma}$ chine Learning, ICLR 2019 Workshop, New Orleans, Louisiana, United States, May 6, 2019. OpenReview.net.

Frank Nielsen. 2020. On a generalization of the jensenshannon divergence and the jensen-shannon centroid. Entropy, 22(2):221. 
Kishore Papineni, Salim Roukos, Todd Ward, and WeiJing Zhu. 2002. Bleu: A method for automatic evaluation of machine translation. In Proceedings of the 40th Annual Meeting on Association for Computational Linguistics, ACL '02, pages 311-318, Stroudsburg, PA, USA. Association for Computational Linguistics.

Michael Tschannen, Olivier Frederic Bachem, and Mario Lučić. 2018. Recent advances in autoencoderbased representation learning. In Bayesian Deep Learning Workshop, NeurIPS.

Peng Xu, Yanshuai Cao, and Jackie Chi Kit Cheung. 2020. On variational learning of controllable representations for text without supervision. In 37th International Conference on Machine Learning, ICML.

Zichao Yang, Zhiting Hu, Chris Dyer, Eric P Xing, and Taylor Berg-Kirkpatrick. 2018. Unsupervised text style transfer using language models as discriminators. In S. Bengio, H. Wallach, H. Larochelle, K. Grauman, N. Cesa-Bianchi, and R. Garnett, editors, Advances in Neural Information Processing Systems 31, pages 7287-7298. Curran Associates, Inc.

Kaizhong Zhang and Dennis E. Shasha. 1989. Simple fast algorithms for the editing distance between trees and related problems. SIAM J. Comput., 18(6):1245-1262.

Qingchen Zhang, Laurence T Yang, Zhikui Chen, and Peng Li. 2018. A survey on deep learning for big data. Information Fusion, 42:146-157.

Jake Zhao, Yoon Kim, Kelly Zhang, Alexander M Rush, and Yann LeCun. 2018. Adversarially regularized autoencoders. In 35th International Conference on Machine Learning, ICML 2018, pages 9405-9420. International Machine Learning Society (IMLS). 


\section{Appendix}

\section{Polarized-VAE: Proximity Based Disentangled Representation Learning for Text Generation}

\section{A Model and Training Details}

Both the semantic and syntactic encoders are bidirectional LSTMs (Hochreiter and Schmidhuber, 1997) with hidden size of 128 , followed by two feed-forward layers to parameterize the Gaussian mean $(\mu)$ and standard deviation $(\sigma)$ parameters similar to standard VAE formulations used by (Bao et al., 2019). The latent space dimensions were taken to be $\operatorname{dim}\left(z^{1}\right)=64$ and $\operatorname{dim}\left(z^{2}\right)=16$. The decoder is a unidirectional LSTM with a hidden size of 128. We train the model for 30 epochs in total using the ADAM optimizer (Kingma and $\mathrm{Ba}$, 2015) with the default parameters and a learning rate of 0.001 .

We adopt the standard tricks for VAE training including dropout and KL annealing followed by (Bowman et al., 2016). We anneal both semantic and syntactic KL weights $\left(\lambda_{k l}\right)$ upto 0.3 (5000 steps) using the same sigmoid schedule (Bahuleyan, 2018).

\section{B Proximity Functions}

We provide results for the other proximity functions that we explored for the polarized-VAE model.

\begin{tabular}{|c|c|c|c|}
\hline Metric & $\Delta \mathrm{BLEU}^{\uparrow}$ & $\Delta \mathrm{TED}^{\uparrow}$ & $\Delta \mathrm{GM}^{\uparrow}$ \\
\hline Cosine Distance & $\mathbf{9 . 8 6}$ & $\mathbf{2 . 4 2}$ & $\mathbf{4 . 8 8}$ \\
Hellinger Distance & 4.12 & 0.86 & 1.42 \\
MMD & 5.21 & 1.17 & 1.91 \\
KL Divergence & 4.32 & 0.75 & 1.28 \\
JS Divergence & 5.81 & 1.46 & 2.33 \\
\hline
\end{tabular}

Table 2: Comparison of polarized-VAE with different proximity functions.

We note that since there is no closed form expression for the JS divergence between two Normal Random variables we used the generalized JS Divergence proposed by (Nielsen, 2020).

\section{Transfer Examples}

We provide qualitative examples of our transfer experiments, where we generate a sentence with the semantics of $x_{\text {sem }}$ and the syntactic structure of $x_{\text {syn }}$ in Table 4 . We also provide the sentences generated by a standard-VAE for comparison.

\section{Disentanglement of Latent Subspaces}

We test if there a possibility that the two latent subspaces encode similar information. This is only likely to happen if the attributes themselves are highly correlated (e.g., if we want to disentangle syntax from length). For such cases, even existing methods based on adversarial disentanglement (John et al., 2019) may fail to completely separate out correlated information.

However, if the attributes are different enough (or ideally independent) for e.g., syntax and semantics, this is less problematic. Note that we apply our proximity loss independently to each of the subspaces (i.e., leaving the other space(s) untouched for a given input). This encourages the semantic encoder to encode semantically similar sentences close together and dissimilar ones far apart in the semantic space (same applies for the syntax encoder).

We empirically compute correlations between the semantic and syntax latent vectors for 1000 test sentences as a way to check whether the two encoders learn similar information. By feeding 1000 sentences from the test set to the Polarized-VAE, we obtain their corresponding semantic $\left(z_{\mathrm{sem}}\right)$ and $\operatorname{syntax}\left(z_{\text {syn }}\right)$ latent vectors. We then empirically compute the correlation between $z_{\text {sem }}$ and $z_{\text {syn }}$. To analyze the level of similarity of information represented in $z_{\mathrm{sem}}$ and $z_{\mathrm{syn}}$, we report the maximum absolute correlation (max across all pairs of dimensions) and also the mean absolute correlation. A higher value of correlation would indicate that there is more overlapping information learnt by the semantic and syntactic encoders. As illustrated in Table 3, the analysis indicates that the semantic and syntax latent vectors in polarized-VAE encodes less correlated information than standard-VAE (due to the proximity-based regularization). This demonstrates that the 2 latent spaces learned by our model encode sufficiently different information.

\begin{tabular}{|c|c|c|}
\hline Model & Max Abs Corr & Mean Abs Corr \\
\hline standard-VAE & 0.62 & 0.1 \\
polarized-VAE & $\mathbf{0 . 2 5}$ & $\mathbf{0 . 0 5}$ \\
\hline
\end{tabular}

Table 3: Maximum Absolute Correlation and Mean Absolute Correlation between the semantic and syntactic latent vectors. 


\begin{tabular}{l|l|l|l}
\hline \multicolumn{1}{c|}{$\boldsymbol{x}_{\text {sem }}$} & \multicolumn{1}{c|}{$\boldsymbol{x}_{\text {syn }}$} & \multicolumn{1}{c|}{ polarized-VAE } & \multicolumn{1}{c}{ standard-VAE } \\
\hline $\begin{array}{l}\text { A man works near a ve- } \\
\text { hicle. }\end{array}$ & $\begin{array}{l}\text { A woman showing her } \\
\text { face from something to } \\
\text { her friend. }\end{array}$ & $\begin{array}{l}\text { A man directing traffic } \\
\text { on a bicycle to an emer- } \\
\text { gency vehicle. }\end{array}$ & $\begin{array}{l}\text { A woman works on a } \\
\text { loom while sitting out- } \\
\text { side. }\end{array}$ \\
\hline $\begin{array}{l}\text { A family in a party } \\
\text { preparing food and en- } \\
\text { joying a meal. }\end{array}$ & Man reading a book. & $\begin{array}{l}\text { A person enjoying } \\
\text { food. }\end{array}$ & A man plays his guitar. \\
\hline $\begin{array}{l}\text { Two young boys are } \\
\text { standing around a cam- } \\
\text { era outdoors. }\end{array}$ & $\begin{array}{l}\text { Three kids are on stage } \\
\text { with a vacuum cleaner. }\end{array}$ & $\begin{array}{l}\text { Two young boys are } \\
\text { standing around a cam- } \\
\text { era outdoors. }\end{array}$ & $\begin{array}{l}\text { Two people are stand- } \\
\text { ing on a snowy hill. }\end{array}$ \\
\hline $\begin{array}{l}\text { There are a group of peo- } \\
\text { ple sitting down. }\end{array}$ & They are outside. & There are people. & They are outside \\
\hline $\begin{array}{l}\text { a woman wearing a hat } \\
\text { and hat is chopping co- } \\
\text { conuts with machete. }\end{array}$ & $\begin{array}{l}\text { The person is in a blue } \\
\text { shirt playing with a a } \\
\text { ball. }\end{array}$ & $\begin{array}{l}\text { a woman with a hat is } \\
\text { hanging upside down } \\
\text { over utensils. }\end{array}$ & $\begin{array}{l}\text { A girl in a pink shirt } \\
\text { and elbow pads is } \\
\text { swirling bubbles. }\end{array}$ \\
\hline $\begin{array}{l}\text { The young girl and a a } \\
\text { grownup are standing } \\
\text { around a table, in front } \\
\text { of a fence. }\end{array}$ & $\begin{array}{l}\text { A guy stands with cane } \\
\text { outdoors. }\end{array}$ & $\begin{array}{l}\text { The young girl is out- } \\
\text { side. }\end{array}$ & $\begin{array}{l}\text { The little boy is doing } \\
\text { a show. }\end{array}$ \\
\hline $\begin{array}{l}\text { A person is sleeping on } \\
\text { bed. }\end{array}$ & $\begin{array}{l}\text { A man and his son are } \\
\text { walking to the beach } \\
\text { looking for something. }\end{array}$ & $\begin{array}{l}\text { A man and a child sit } \\
\text { on the ground covered } \\
\text { in bed with rocks. }\end{array}$ & $\begin{array}{l}\text { A man is wearing blue } \\
\text { jeans and a blue shirt } \\
\text { walking. }\end{array}$ \\
\hline $\begin{array}{l}\text { The men and women are } \\
\text { enjoying a waterfall. }\end{array}$ & $\begin{array}{l}\text { A dog is holding an ob- } \\
\text { ject. }\end{array}$ & $\begin{array}{l}\text { The man and woman } \\
\text { are outdoors. }\end{array}$ & $\begin{array}{l}\text { The two men are work- } \\
\text { ing on the roof. }\end{array}$ \\
\hline $\begin{array}{l}\text { a man dressed in uni- } \\
\text { form. }\end{array}$ & $\begin{array}{l}\text { There is a man with a } \\
\text { horse on it. }\end{array}$ & $\begin{array}{l}\text { A man dressed in black } \\
\text { clothing works in a } \\
\text { house. }\end{array}$ & $\begin{array}{l}\text { A man dressed in black } \\
\text { and white holding a } \\
\text { baby. }\end{array}$ \\
\hline
\end{tabular}

Table 4: Examples of transferred sentences that use the semantics of $x_{\text {sem }}$ and syntax of $x_{\text {syn }}$ 